DOI: : 10.1016/j.jde.2017.12.022

\title{
NONLINEAR SECOND ORDER EVOLUTION INCLUSIONS WITH NONCOERCIVE VISCOSITY TERM
}

\author{
NIKOLAOS S. PAPAGEORGIOU, VICENŢIU D. RĂDULESCU, AND DUŠAN D. REPOVŠ
}

\begin{abstract}
In this paper we deal with a second order nonlinear evolution inclusion, with a nonmonotone, noncoercive viscosity term. Using a parabolic regularization (approximation) of the problem and a priori bounds that permit passing to the limit, we prove that the problem has a solution.
\end{abstract}

\section{INTRODUCTION}

Let $T=[0, b]$ and let $\left(X, H, X^{*}\right)$ be an evolution triple of spaces, with the embedding of $X$ into $H$ being compact (see Section 2 for definitions).

In this paper, we study the following nonlinear evolution inclusion:

$$
\left\{\begin{array}{l}
u^{\prime \prime}(t)+A\left(t, u^{\prime}(t)\right)+B u(t) \in F\left(t, u(t), u^{\prime}(t)\right) \text { for almost all } t \in T, \\
u(0)=u_{0}, u^{\prime}(0)=u_{1}
\end{array}\right\}
$$

In the past, such multi-valued problems were studied by Gasinski [3], Gasinski and Smolka [6, 7], Migórski et al. [11, 12, 13, 14], Ochal [15], Papageorgiou, Rădulescu and Repovš [16, 17], Papageorgiou and Yannakakis [18, 19]. The works of Gasinski [3], Gasinski and Smolka [6, 7] and Ochal [15], all deal with hemivariational inequalities, that is, $F(t, x, y)=\partial J(x)$ with $J(\cdot)$ being a locally Lipschitz functional and $\partial J(\cdot)$ denoting the Clarke subdifferential of $J(\cdot)$. In Papageorgiou and Yannakakis $[18,19]$, the multivalued term $F(t, x, y)$ is general (not necessarily of the subdifferential type) and depends also on the time derivative of the unknown function $u(\cdot)$. With the exception of Gasinski and Smolka [7], in all the other works the viscosity term $A(t, \cdot)$ is assumed to be coercive or zero. In the work of Gasinski and Smolka [7], the viscosity term is autonomous (that is, time independent) and $A: X \rightarrow X^{*}$ is linear and bounded.

In this work, the viscosity term $A: T \times X \rightarrow X^{*}$ is time dependent, noncoercive, nonlinear and nonmonotone in $x \in X$. In this way, we extend and improve the result of Gasinski and Smolka [7]. Our approach uses a kind of parabolic regularization of the inclusion, analogous to the one used by Lions [10, p. 346] in the context of semilinear hyperbolic equations.

\section{Mathematical Background and Hypotheses}

Let $V, Y$ be Banach spaces and assume that $V$ is embedded continuously and densely into $Y$ (denoted by $V \hookrightarrow Y$ ). Then we have the following properties:

(i) $Y^{*}$ is embedded continuously into $V^{*}$;

(ii) if $V$ is reflexive, then $Y^{*} \hookrightarrow V^{*}$.

Key words and phrases. Evolution triple, compact embedding, parabolic regularization, noncoercive viscosity term, a priori bounds.

2010 AMS Subject Classification: 35L90 (Primary); 35R70, 47H04, 47H05 (Secondary). 
The following notion is a useful tool in the theory of evolution equations.

Definition 1. By an "evolution triple" (or "Gelfand triple") we understand a triple of spaces $\left(X, H, X^{*}\right)$ such that

(a) $X$ is a separable reflexive Banach space and $X^{*}$ is its topological dual;

(b) $H$ is a separable Hilbert space identified with its dual $H^{*}$, that is, $H=H^{*}$ (pivot space);

(c) $X \hookrightarrow H$.

Then from the initial remarks we have

$$
X \hookrightarrow H=H^{*} \hookrightarrow X^{*} .
$$

In what follows, we denote by $\|\cdot\|$ the norm of $X$, by $|\cdot|$ the norm of $H$ and by $\|\cdot\|_{*}$ the norm of $X^{*}$. Evidently we can find $\hat{c}_{1}, \hat{c}_{2}>0$ such that

$$
|\cdot| \leqslant \hat{c}_{1}|| \cdot|| \text { and }\|\cdot\|_{*} \leqslant \hat{c}_{2}|\cdot| \text {. }
$$

By $(\cdot, \cdot)$ we denote the inner product of $H$ and by $\langle\cdot, \cdot\rangle$ the duality brackets for the pair $\left(X^{*}, X\right)$. We have

$$
\left.\langle\cdot, \cdot\rangle\right|_{H \times X}=(\cdot, \cdot) .
$$

Let $1<p<\infty$. The following space is important in the study of problem (1):

$$
W_{p}(0, b)=\left\{u \in L^{p}(T, X): u^{\prime} \in L^{p^{\prime}}\left(T, X^{*}\right)\right\}\left(\frac{1}{p}+\frac{1}{p^{\prime}}=1\right) .
$$

Here $u^{\prime}$ is understood in the distributional sense (weak derivative). We know that $L^{p}(T, X)^{*}=L^{p^{\prime}}\left(T, X^{*}\right)$ (see, for example, Gasinski and Papageorgiou [4, p. 129]). Suppose that $u \in W_{p}(0, b)$. If we view $u(\cdot)$ as an $X^{*}$-valued function, then $u(\cdot)$ is absolutely continuous, hence differentiable almost everywhere and this derivative coincides with the distributional one. So, $u^{\prime} \in L^{p^{\prime}}\left(T, X^{*}\right)$ and we can say

$$
W_{p}(0, b) \subseteq A C^{1, p^{\prime}}\left(T, X^{*}\right)=W^{1, p^{\prime}}\left((0, b), X^{*}\right) .
$$

The space $W_{p}(0, b)$ is equipped with the norm

$$
\|u\|_{W_{p}}=\left[\|u\|_{L^{p}(T, X)}^{p}+\left\|u^{\prime}\right\|_{L^{p^{\prime}}\left(T, X^{*}\right)}^{p}\right]^{\frac{1}{p}} \text { for all } u \in W_{p}(0, b) .
$$

Evidently, another equivalent norm on $W_{p}(0, b)$ is

$$
|u|_{W_{p}}=\|u\|_{L^{p}(T, X)}+\left\|u^{\prime}\right\|_{L^{p}\left(T, X^{*}\right)} \text { for all } u \in W_{p}(0, b) .
$$

With any of the above norms, $W_{p}(0, b)$ becomes a separable reflexive Banach space. We have that

$$
\begin{aligned}
& W_{p}(0, b) \hookrightarrow C(T, H) ; \\
& W_{p}(0, b) \hookrightarrow L^{p}(T, H) \text { and the embedding is compact. }
\end{aligned}
$$

The elements of $W_{p}(0, b)$ satisfy an integration by parts formula which will be useful in our analysis.

Proposition 2. If $u, v \in W_{p}(0, b)$ and $\xi(t)=(u(t), v(t))$ for all $t \in T$, then $\xi(\cdot)$ is absolutely continuous and $\frac{d \xi}{d t}(t)=\left\langle u^{\prime}(t), v(t)\right\rangle+\left\langle u(t), v^{\prime}(t)\right\rangle$ for almost all $t \in T$. 
Now suppose that $(\Omega, \Sigma, \mu)$ is a finite measure space, $\Sigma$ is $\mu$-complete and $Y$ is a separable Banach space. A multifunction (set-valued function) $F: \Omega \rightarrow 2^{Y} \backslash\{\emptyset\}$ is said to be "graph measurable", if

$$
\operatorname{Gr} F=\{(\omega, y) \in \Omega \times Y: y \in F(\omega)\} \in \Sigma \times B(Y),
$$

with $B(Y)$ being the Borel $\sigma$-field of $Y$.

If $F(\cdot)$ has closed values, then graph measurability is equivalent to saying that for every $y \in Y$ the $\mathbb{R}_{+}$-valued function

$$
\omega \mapsto d(y, F(\omega))=\inf \left\{\|y-v\|_{Y}: v \in F(\omega)\right\}
$$

is $\Sigma$-measurable.

Given a graph measurable multifunction $F: \Omega \rightarrow 2^{Y} \backslash\{\emptyset\}$, the Yankov-von Neumann-Aumann selection theorem (see Hu and Papageorgiou [8, p. 158]) implies that $F(\cdot)$ admits a measurable selection, i.e. that there exists $f: \Omega \rightarrow Y$ a $\Sigma$ measurable function such that $f(\omega) \in F(\omega) \mu$-almost everywhere. In fact, we can find an entire sequence $\left\{f_{n}\right\}_{n \geqslant 1}$ of measurable selections such that $F(\omega) \subseteq$ ${\overline{\left\{f_{n}(\omega)\right\}_{n} \geqslant 1}} \mu$-almost everywhere.

For $1 \leqslant p \leqslant \infty$, we define

$$
S_{F}^{p}=\left\{f \in L^{p}(\Omega, Y): f(\omega) \in F(\omega) \mu \text {-almost everywhere }\right\} .
$$

It is easy to see that $S_{F}^{p} \neq \emptyset$ if and only if $\omega \mapsto \inf \left\{\|v\|_{Y}: v \in F(\omega)\right\}$ belongs to $L^{p}(\Omega)$. This set is "decomposable" in the sense that if $\left(A, f_{1}, f_{2}\right) \in \Sigma \times S_{F}^{p} \times S_{F}^{p}$, then

$$
\chi_{A} f_{1}+\chi_{A^{c}} f_{2} \in S_{F}^{p} .
$$

Finally, for a sequence $\left\{C_{n}\right\}_{n \geqslant 1}$ of nonempty subsets of $Y$, we define $w-\limsup _{n \rightarrow \infty} C_{n}=\left\{y \in Y: y=w-\lim _{k \rightarrow \infty} y_{n_{k}}, y_{n_{k}} \in C_{n_{k}}, n_{1}<n_{2}<\cdots<n_{k}<\cdots\right\}$.

For more details on the notions discussed in this section, we refer to Gasinski and Papageorgiou [4], Roubiček [20], Zeidler [21] (for evolution triples and related notations) and $\mathrm{Hu}$ and Papageorgiou [8] (for measurable multifunctions).

Let $V$ be a reflexive Banach space and $A: V \rightarrow V^{*}$ a map. We say that $A$ is "pseudomonotone", if $A$ is continuous from every finite dimensional subspace of $V$ into $V_{w}^{*}$ (= the dual $V^{*}$ equipped with the weak topology) and if

$$
v_{n} \stackrel{w}{\rightarrow} v \text { in } V, \limsup _{n \rightarrow \infty}\left\langle A\left(v_{n}\right), v_{n}-v\right\rangle \leqslant 0
$$

then

$$
\langle A(v), v-y\rangle \leqslant \liminf _{n \rightarrow \infty}\left\langle A\left(v_{n}\right), v_{n}-y\right\rangle \text { for all } y \in V .
$$

An everywhere defined maximal monotone operator is pseudomonotone. If $V$ is finite dimensional, then every continuous map $A: V \rightarrow V^{*}$ is pseudomonotone.

In what follows, for any Banach space $Z$, we will use the following notations:

$$
\begin{aligned}
& P_{f(c)}(Z)=\{C \subseteq Z: C \text { is nonempty, closed (and convex) }\}, \\
& P_{(w) k(c)}(Z)=\{C \subseteq Z: C \text { is nonempty, (weakly-) compact (and convex) }\} .
\end{aligned}
$$

The hypotheses on the data of problem (1) are the following:

$H(A): A: T \times T \rightarrow X^{*}$ is a map such that

(i) for all $y \in X, t \mapsto A(t, y)$ is measurable;

(ii) for almost all $t \in T$, the map $y \mapsto A(t, y)$ is pseudomonotone; 
(iii) $\|A(t, y)\|_{*} \leqslant a_{1}(t)+c_{1}\|y\|^{p-1}$ for almost all $t \in T$ and all $y \in X$, with $a_{1} \in L^{p^{\prime}}(T), c_{1}>0,2 \leqslant p<\infty ;$

(iv) $\langle A(t, y), y\rangle \geqslant 0$ for almost all $t \in T$ and all $y \in X$.

$H(B): B \in \mathscr{L}\left(X, X^{*}\right),\langle B x, y\rangle=\langle x, B y\rangle$ for all $x, y \in X$ and $\langle B x, x\rangle \geqslant c_{0}\|x\|^{2}$ for all $x \in X$ and some $c_{0}>0$.

$H(F): F: T \times H \times H \rightarrow P_{f_{c}}(H)$ is a multifunction such that

(i) for all $x, y \in H, t \mapsto F(t, x, y)$ is graph measurable;

(ii) for almost all $t \in T$, the graph $\operatorname{Gr} F(t, \cdot, \cdot)$ is sequentially closed in $H \times$ $H_{w} \times H_{w}$ (here $H_{w}$ denotes the Hilbert space $H$ furnished with the weak topology);

(iii) $|F(t, x, y)|=\sup \{|h|: h \in F(t, x, y)\} \leqslant a_{2}(t)(1+|x|+|y|)$ for almost all $t \in T$ and all $x, y \in H$ with $a_{2} \in L^{2}(T)_{+}$.

Definition 3. We say that $u \in C(T, X)$ is a "solution" of problem (1) with $u_{0} \in$ $X, u_{1} \in H$, if

- $u^{\prime} \in W_{p}(0, b)$ and

- there exists $f \in S_{F\left(\cdot, u(\cdot), u^{\prime}(\cdot)\right)}^{2}$ such that

$$
\left\{\begin{array}{l}
u^{\prime \prime}(t)+A\left(t, u^{\prime}(t)\right)+B u(t)=f(t) \text { for almost all } t \in T, \\
u(0)=u_{0}, u^{\prime}(0)=u_{1} .
\end{array}\right\}
$$

In what follows, we denote by $S\left(u_{0}, u_{1}\right)$ the set of solutions of problem (1). Recalling that $W_{p}(0, b) \hookrightarrow C(T, H)$ (see $\left.(3)\right)$, we have that

$$
S\left(u_{0}, u_{1}\right) \subseteq C^{1}(T, H)
$$

By Troyanski's renorming theorem (see Gasinski and Papageorgiou [4, p. 911]) we may assume without loss of generality that both $X$ and $X^{*}$ are locally uniformly convex. Let $\mathcal{F}: X \rightarrow X^{*}$ be the duality map of $X$ defined by

$$
\mathcal{F}(x)=\left\{x^{*} \in X^{*}:\left\langle x^{*}, x\right\rangle=\|x\|^{2}=\left\|x^{*}\right\|_{*}^{2}\right\} .
$$

We know that $\mathcal{F}(\cdot)$ is single-valued and a homeomorphism (see Gasinski and Papageorgiou $[4$, p. 316] and Zeidler [21, p. 861]).

For every $r \geqslant p$, let $K_{r}: X \rightarrow X^{*}$ be the map defined by

$$
K_{r}(y)=\|y\|^{r-2} \mathcal{F}(y) \text { for all } y \in X \text {. }
$$

\section{Existence TheOrem}

Given $\epsilon>0$, we consider the following perturbation (parabolic regularization) of problem (1):

$$
\left\{\begin{array}{l}
u^{\prime \prime}(t)+A\left(t, u^{\prime}(t)\right)+\epsilon K_{r}\left(u^{\prime}(t)\right)+B u(t) \in F\left(t, u(t), u^{\prime}(t)\right) \text { for a.a. } t \in T, \\
u(0)=u_{0}, u^{\prime}(0)=u_{1} .
\end{array}\right\}
$$

Consider the map $A_{\epsilon}: T \times X \rightarrow X^{*}$ defined by

$$
A_{\epsilon}(t, y)=A(t, y)+\epsilon K_{r}(y) \text { for all } t \in T \text {, and all } y \in X .
$$

This map has the following properties:

(i) for all $y \in X$, the map $t \mapsto A_{\epsilon}(t, y)$ is measurable;

(ii) for almost all $t \in T$, the map $y \mapsto A_{\epsilon}(t, y)$ is pseudomonotone;

(iii) $\left\|A_{\epsilon}(t, y)\right\|_{*} \leqslant \hat{a}_{1}(t)+\hat{c}_{1}\|y\|^{r-1}$ for almost all $t \in T$, all $y \in X$ and with $\hat{a}_{1} \in L^{p^{\prime}}(T), \hat{c}_{1}>0$ (recall that $r \geqslant p$ and $\frac{1}{r}+\frac{1}{r^{\prime}}=1$ ); 
(iv) $\left\langle A_{\epsilon}(t, y), y\right\rangle \geqslant \epsilon\|y\|^{r}$ for all $t \in T$, all $y \in X$.

So, in problem (1) the viscosity term $A_{\epsilon}(t, \cdot)$ is coercive. Therefore we can apply Theorem 1 of Papageorgiou and Yannakakis [18] and we obtain the following existence result for the approximate (regularized) problem (5).

Proposition 4. If hypotheses $H(A), H(B), H(F)$ hold and $u_{0} \in X, u_{1} \in H$, then problem (5) admits a solution $u_{\epsilon} \in W^{1, r}((0, b), X) \cap C^{1}(T, H)$ with

$$
u_{\epsilon}^{\prime} \in W_{r}(0, b) .
$$

To produce a solution for the original problem (1), we have to pass to the limit as $\epsilon \rightarrow 0^{+}$. To do this, we need to have a priori bounds for the solutions $u_{\epsilon}(\cdot)$ which are independent of $\epsilon \in(0,1]$ and $r \geqslant p$.

Proposition 5. If hypotheses $H(A), H(B), H(F)$ hold, $u_{0} \in X, u_{1} \in H$ and $u(\cdot)$ is a solution of (5), then there exists $M_{0}>0$ which is independent of $\epsilon \in(0,1]$ and $r \geqslant p$ for which we have

$$
\|u\|_{C(T, X)},\left\|u^{\prime}\right\|_{C(T, H)}, \epsilon^{\frac{1}{r}}\left\|u^{\prime}\right\|_{L^{r}(T, X)},\left\|u^{\prime \prime}\right\|_{L^{2}\left(T, X^{*}\right)} \leqslant M_{0} .
$$

Proof. It follows from Proposition 4 that $u^{\prime} \in W_{r}(0, b)$ and that there exists $f \in$ $S_{F\left(\cdot, u(\cdot), u^{\prime}(\cdot)\right)}^{2}$ such that

$$
u^{\prime \prime}(t)+A\left(t, u^{\prime}(t)\right)+\epsilon K_{r}\left(u^{\prime}(t)\right)+B u(t)=f(t) \text { for almost all } t \in T .
$$

We act with $u^{\prime}(t) \in X$. Then

$$
\left\langle u^{\prime \prime}(t), u^{\prime}(t)\right\rangle+\left\langle A\left(t, u^{\prime}(t)\right), u^{\prime}(t)\right\rangle+\epsilon\left\langle K_{r}\left(u^{\prime}(t)\right), u^{\prime}(t)\right\rangle=\left(f(t), u^{\prime}(t)\right)
$$

for almost all $t \in T$ (see (2)).

We examine separately each summand on the left-hand side of (6). Recall that $u_{r}^{\prime} \in W_{r}(0, b)$. So from Proposition 2 (the integration by parts formula), we have

$$
\left\langle u^{\prime \prime}(t), u^{\prime}(t)\right\rangle=\frac{1}{2} \frac{d}{d t}\left|u^{\prime}(t)\right|^{2} \text { for almost all } t \in T .
$$

Hypothesis $H(A)(i v)$ and the definition of the duality map, imply that

$$
\left\langle A\left(t, u^{\prime}(t)\right), u^{\prime}(t)\right\rangle+\epsilon\left\langle K_{r}\left(u^{\prime}(t)\right), u^{\prime}(t)\right\rangle \geqslant \epsilon\left\|u^{\prime}(t)\right\|^{r} \text { for almost all } t \in T .
$$

By hypothesis $H(B)$, we have

$$
\left\langle B u(t), u^{\prime}(t)\right\rangle=\frac{1}{2} \frac{d}{d t}\langle B u(t), u(t)\rangle \text { for almost all } t \in T .
$$

We return to (6) and use (7), (8), (9). We obtain

$$
\begin{aligned}
& \frac{1}{2} \frac{d}{d t}\left|u^{\prime}(t)\right|_{2}+\epsilon\left\|u^{\prime}(t)\right\|^{r}+\frac{1}{2} \frac{d}{d t}\langle B u(t), u(t)\rangle \leqslant\left(f(t), u^{\prime}(t)\right) \text { for a.a. } t \in T, \\
\Rightarrow & \frac{1}{2}\left|u^{\prime}(t)\right|^{2}+\epsilon \int_{0}^{t}\left\|u^{\prime}(s)\right\|^{r} d s+c_{0}\|u(t)\|^{2} \\
(10) \leqslant & \left.\int_{0}^{t}\left(f(s), u^{\prime}(s)\right) d s+\frac{1}{2}\left|u_{1}\right|^{2}+\frac{1}{2}\|B\| \mathscr{L}\left\|u_{0}\right\|^{2} \text { (see hypothesis } H(B)\right) .
\end{aligned}
$$


Using hypothesis $H(F)(i i i)$, we get

$$
\begin{aligned}
& \int_{0}^{t}\left(f(s), u^{\prime}(s)\right) d s \\
\leqslant & \int_{0}^{t}\left[a_{2}(s)+a_{2}(s)\left(|u(s)|+\left|u^{\prime}(s)\right|\right)\right]\left|u^{\prime}(s)\right| d s \\
\leqslant & \int_{0}^{t}\left|u^{\prime}(s)\right|^{2} d s+\int_{0}^{t} a_{2}(s)^{2} d s+\int_{0}^{t} a_{2}(s)^{2}\left[|u(s)|^{2}+\left|u^{\prime}(s)\right|^{2}\right] d s .
\end{aligned}
$$

Recall that $u \in W^{1, r}((0, b), X)$ (see Proposition 4). So, $u \in A C^{1, r}(T, H)$ and we can write

$$
u(t)=u_{0}+\int_{0}^{t} u^{\prime}(s) d s \text { for all } t \in T
$$

(12户 $|u(t)|^{2} \leqslant 2\left|u_{0}\right|^{2}+2 b \int_{0}^{t}\left|u^{\prime}(s)\right|^{2} d s$ for all $t \in T$ (using Jensen's inequality).

We use (12) in (11) and obtain

$$
\begin{aligned}
& \int_{0}^{t}\left(f(s), u^{\prime}(s)\right) d s \\
\leqslant & \left\|a_{2}\right\|_{2}^{2}+\int_{0}^{t}\left[1+a_{2}(s)^{2}\right]\left|u^{\prime}(s)\right|^{2} d s+\int_{0}^{t} 2 a_{2}(s)^{2}\left[\left|u_{0}\right|^{2}+b \int_{0}^{s}\left|u^{\prime}(\tau)\right|^{2} d \tau\right] d s \\
(13 \Varangle \quad & c_{2}+\int_{0}^{t} \eta(s)\left|u^{\prime}(s)\right|^{2} d s+2 b \int_{0}^{t} a_{2}(s)^{2} \int_{0}^{s}\left|u^{\prime}(\tau)\right|^{2} d \tau d s \\
& \text { for some } c_{2}>0 \text { and } \eta \in L^{1}(T) .
\end{aligned}
$$

We use (13) in (10) and have

$$
\begin{aligned}
& \frac{1}{2}\left|u^{\prime}(t)\right|^{2}+\epsilon \int_{0}^{t}\left\|u^{\prime}(s)||^{p} d s+c_{0}\right\| u(t) \|^{2} \\
\leqslant & c_{3}+\int_{0}^{t} \eta(s)\left|u^{\prime}(s)\right|^{2} d s+2 b \int_{0}^{t} a_{2}(s)^{2} \int_{0}^{s}\left|u^{\prime}(\tau)\right|^{2} d \tau d s \text { for some } c_{3}>0 .
\end{aligned}
$$

Invoking Proposition 1.7.87 of Denkowski, Migórski and Papageorgiou [2, p. 128] we can find $M>0$ (independent of $\epsilon \in(0,1]$ and $r \geqslant p)$ such that

$$
\begin{aligned}
& \left|u^{\prime}(t)\right|^{2} \leqslant M \text { for all } t \in T, \\
\Rightarrow \quad & || u^{\prime} \|_{C(T, H)} \leqslant M_{1}=M^{\frac{1}{2}} .
\end{aligned}
$$

Using this bound in (14), we can find $M_{2}>0$ (independent of $\epsilon \in(0,1]$ and $r \geqslant p$ ) such that

$$
\|u\|_{C(T, X)} \leqslant M_{2} \text { and } \epsilon^{\frac{1}{r}}\left\|u^{\prime}\right\|_{L^{r}(T, X)} \leqslant M_{2} .
$$

Finally, directly from (5), we see that there exists $M_{3}>0$ (independent of $\epsilon \in(0,1]$ and $r \geqslant p)$ such that

$$
\left\|u^{\prime \prime}\right\|_{L^{r^{\prime}}}\left(T, X^{*}\right) \leqslant M_{3} .
$$

We set $M_{0}=\max \left\{M_{1}, M_{2}, M_{3}\right\}>0$ and get the desired bound.

The bounds produced in Proposition 5 permit passing to the limit as $\epsilon \rightarrow 0^{+}$to produce a solution for problem (1). 
Theorem 6. If hypotheses $H(A), H(B), H(F)$ hold and $u_{0} \in X, u_{1} \in H$, then $S\left(u_{0}, u_{1}\right) \neq \emptyset$.

Proof. Let $\epsilon_{n} \rightarrow 0^{+}$and let $u_{n}=u_{\epsilon_{n}}$ be solutions of the "regularized" problem (5) (see Proposition 4). Because of the bounds established in Proposition 5 and by passing to a suitable subsequence if necessary, we can say that

$$
\left\{\begin{array}{l}
u_{n} \stackrel{w^{*}}{\longrightarrow} u \text { in } L^{\infty}(T, X), u_{n} \stackrel{w}{\rightarrow} u \text { in } C(T, H), u_{n} \rightarrow u \text { in } L^{r}(T, H) \\
u_{n}^{\prime} \stackrel{w^{*}}{\longrightarrow} y \text { in } L^{\infty}(T, H), u_{n}^{\prime \prime} \stackrel{w}{\rightarrow} v \text { in } L^{r^{\prime}}\left(T, X^{*}\right) \text { (see (3) and (4)). }
\end{array}\right\}
$$

Recall that $u_{n} \in A C^{1, r}(T, H)$ for all $n \in \mathbb{N}$ and so

$$
\begin{aligned}
& u_{n}(t)=u_{0}+\int_{0}^{t} u_{n}^{\prime}(s) d s \text { for all } t \in T, \\
\Rightarrow & u(t)=u_{0}+\int_{0}^{t} y(s) d s \text { for all } t \in T(\text { see }(15)), \\
\Rightarrow & u \in A C^{1, r}(T, H) \text { and } u^{\prime}=y .
\end{aligned}
$$

Since $u_{n} \in W_{r}(0, b)$ for all $n \in \mathbb{N}$, we have

$$
v=y^{\prime}=u^{\prime \prime} \in L^{r^{\prime}}\left(T, X^{*}\right) \text { (see Hu and Papageorgiou [9, p. 6]). }
$$

Let $a: L^{r}(T, X) \rightarrow L^{r^{\prime}}\left(T, X^{*}\right)$ be the nonlinear map defined by

$$
a(u)(\cdot)=A(\cdot, u(\cdot)) \text { for all } u \in L^{r}(T, X) .
$$

Also, let $\hat{K}_{r}: L^{r}(T, X) \rightarrow L^{r^{\prime}}\left(T, X^{*}\right)$ be defined by

$$
\hat{K}_{r}(u)(\cdot)=\|u(\cdot)\|^{r-2} \mathscr{F}(u(\cdot)) \text { for all } u \in L^{r}(T, X) .
$$

Both maps are continuous and monotone, hence maximal monotone (see Gasinski and Papageorgiou [4, Corollary 3.2.32, p. 320]).

Finally, let $\hat{B} \in \mathscr{L}\left(L^{r}(T, X), L^{r^{\prime}}\left(T, X^{*}\right)\right)$ be defined by

$$
\hat{B}(u)(\cdot)=B(u(\cdot)) \text { for all } u \in L^{r}(T, X) .
$$

We have

$$
\begin{array}{r}
u_{n}^{\prime \prime}+a\left(u_{n}^{\prime}\right)+\epsilon_{n} \hat{K}_{r}\left(u_{n}^{\prime}\right)+\hat{B} u_{n}=f_{n} \text { in } L^{r}\left(T, X^{*}\right) \\
\text { with } f_{n} \in S_{F\left(\cdot, u_{n}(\cdot), u_{n}^{\prime}(\cdot)\right)}^{2} \text { for all } n \in \mathbb{N} .
\end{array}
$$

From (15) we have

$$
\begin{aligned}
& u_{n} \stackrel{w}{\rightarrow} u \text { in } L^{r}(T, X), \\
& \Rightarrow \quad \hat{B} u_{n} \stackrel{w}{\rightarrow} \hat{B} u \text { in } L^{r^{\prime}}\left(T, X^{*}\right) \text { as } n \rightarrow \infty .
\end{aligned}
$$

Also, we have

$$
\begin{aligned}
& \left\|\hat{K}_{r}\left(u_{n}^{\prime}\right)\right\|_{L^{r^{\prime}}\left(T, X^{*}\right)}=\left\|u_{n}^{\prime}\right\|_{L^{r}(T, X)}^{r-1}, \\
\Rightarrow \quad & \epsilon_{n}\left\|\hat{K}_{r}\left(u_{n}^{\prime}\right)\right\|_{L^{r^{\prime}}\left(T, X^{*}\right)}=\epsilon_{n}^{\frac{1}{r}}\left(\epsilon_{n}^{\frac{1}{r}}\left\|u_{n}^{\prime}\right\|_{L^{r}(T, X)}\right)^{r-1}\left(\text { recall that } \frac{1}{r}+\frac{1}{r^{\prime}}=1\right) \\
& \leqslant \epsilon_{n}^{\frac{1}{r}} M_{0}^{r-1} \text { for all } n \in \mathbb{N}(\text { see Proposition } 5) \\
(18) \Rightarrow \quad & \epsilon_{n}\left\|\hat{K}_{r}\left(u_{r}^{\prime}\right)\right\|_{L^{r^{\prime}}\left(T, X^{*}\right)} \rightarrow 0 \text { as } n \rightarrow \infty
\end{aligned}
$$

From (15) and since $v=u^{\prime \prime}$, we have

$$
u_{n}^{\prime \prime} \stackrel{w}{\rightarrow} u^{\prime \prime} \text { in } L^{r^{\prime}}\left(T, X^{*}\right) .
$$


Finally, hypothesis $H(F)(i i i)$ and Proposition 5 imply that

$$
\left\{f_{n}\right\}_{n \geqslant 1} \subseteq L^{2}(T, H) \text { is bounded. }
$$

By passing to a subsequence if necessary, we may assume that

$$
f_{n} \stackrel{w}{\rightarrow} f \text { in } L^{2}(T, H) .
$$

Invoking Proposition 3.9 of $\mathrm{Hu}$ and Papageorgiou [8, p. 694], we have

$$
\begin{aligned}
f(t) & \in \overline{\operatorname{conv}} w-\limsup _{n \rightarrow \infty}\left\{f_{n}(t)\right\} \\
& \leqslant \overline{\operatorname{conv}} w-\limsup _{n \rightarrow \infty} F\left(t, u_{n}(t), u_{n}^{\prime}(t)\right) \text { for almost all } t \in T \text { (see (16)). }
\end{aligned}
$$

From (15) we see that

$$
u_{n}^{\prime} \stackrel{w}{\rightarrow} u^{\prime} \text { in } W^{1, r^{\prime}}\left((0, b), X^{*}\right) .
$$

Recall that $W^{1, r^{\prime}}\left((0, b), X^{*}\right) \hookrightarrow C\left(T, X^{*}\right)$. So, it follows that

$$
\begin{aligned}
& u_{n}^{\prime} \stackrel{w}{\rightarrow} u^{\prime} \text { in } C\left(T, X^{*}\right) \\
\Rightarrow \quad & u_{n}^{\prime}(t) \stackrel{w}{\rightarrow} u^{\prime}(t) \text { in } X^{*} \text { for all } t \in T .
\end{aligned}
$$

On the other hand, by Proposition 5 we have

$$
\left|u_{n}^{\prime}(t)\right| \leqslant M_{0} \text { for all } t \in T \text {, all } n \in \mathbb{N} .
$$

So, by passing to a subsequence ( $a$ priori the subsequence depends on $t \in T$ ), we have

$$
\begin{aligned}
& u_{n}^{\prime}(t) \stackrel{w}{\rightarrow} \hat{y}(t) \text { in } H \\
\Rightarrow \quad & \hat{y}(t)=u^{\prime}(t) \text { for all } t \in T(\text { see }(21)) .
\end{aligned}
$$

Hence for the original sequence we have

$$
u_{n}^{\prime}(t) \stackrel{w}{\rightarrow} u^{\prime}(t) \text { in } H \text { for all } t \in T .
$$

We know that $\left\{u_{n}\right\}_{n \geqslant 1} \subseteq W_{r}(0, b)$ is bounded (see Proposition 5 ) and recall that $W_{r}(0, b) \hookrightarrow L^{r}(T, H)$ compactly (see (4)). From this compact embedding and from (22), we obtain

$$
u_{n}(t) \rightarrow u(t) \text { in } H \text { for all } t \in T \text { as } n \rightarrow \infty .
$$

From (20), (22), (23) and hypothesis $H(F)(i i i)$ we infer that

$$
\begin{aligned}
& f(t) \in F\left(t, u(t), u^{\prime}(t)\right) \text { for almost all } t \in T, \\
\Rightarrow \quad & f \in S_{F\left(\cdot, u(\cdot), u^{\prime}(\cdot)\right)}^{2} .
\end{aligned}
$$

In what follows, we denote by $((\cdot, \cdot))$ the duality brackets for the pair

$$
\left(L^{r}\left(T, X^{*}\right), L^{r}(T, X)\right) .
$$

Acting with $u_{n}^{\prime}-u^{\prime} \in L^{r}(T, X)$ on (16), we have

$$
\begin{aligned}
& \left(\left(u_{n}^{\prime \prime}, u_{n}^{\prime}-u^{\prime}\right)\right)+\left(\left(a\left(u_{n}^{\prime}\right), u_{n}^{\prime}-u^{\prime}\right)\right)+\left(\left(\epsilon_{n} \hat{K}_{r}\left(u_{n}^{\prime}\right), u_{r}^{\prime}-u^{\prime}\right)\right)+\left(\left(\hat{B} u_{n}, u_{n}^{\prime}-u^{\prime}\right)\right) \\
(24)= & \int_{0}^{b}\left(f_{n}, u_{n}^{\prime}-u^{\prime}\right) d t \text { for all } n \in \mathbb{N} .
\end{aligned}
$$


Note that

$$
\begin{aligned}
\left(\left(u_{n}^{\prime \prime}, u_{n}^{\prime}-u^{\prime}\right)\right)= & \int_{0}^{b}\left\langle u_{n}^{\prime \prime}, u_{n}^{\prime}-u^{\prime}\right\rangle d t \\
= & \int_{0}^{b}\left\langle u_{n}^{\prime \prime}-u^{\prime \prime}, u_{n}^{\prime}-u^{\prime}\right\rangle d t+\left(\left(u^{\prime \prime}, u_{n}^{\prime}-u^{\prime}\right)\right) \\
= & \int_{0}^{b} \frac{1}{2} \frac{d}{d t}\left|u_{n}^{\prime}-u^{\prime}\right|^{2} d t+\left(\left(u^{\prime \prime}, u_{n}^{\prime}-u^{\prime}\right)\right) \text { (see Proposition 2) } \\
= & \frac{1}{2}\left|u_{n}^{\prime}(b)-u^{\prime}(b)\right|^{2}+\left(\left(u^{\prime \prime}, u_{n}^{\prime}-u^{\prime}\right)\right) \\
& \left(\operatorname{since} u_{n}^{\prime}(0)=u^{\prime}(0)=u_{1} \text { for all } n \in \mathbb{N}, \text { see }(22)\right) \\
\Rightarrow & \liminf _{n \rightarrow \infty}\left(\left(u_{n}^{\prime \prime}, u_{n}^{\prime}-u^{\prime}\right)\right)=\frac{1}{2} \liminf _{n \rightarrow \infty}\left|u_{n}^{\prime}(b)-u^{\prime}(b)\right|^{2} \geqslant 0 .
\end{aligned}
$$

Also we have

$$
\begin{aligned}
&\left(\left(\hat{B}\left(u_{n}-u\right), u_{n}^{\prime}-u^{\prime}\right)\right)=\int_{0}^{b} \frac{1}{2} \frac{d}{d t}\left\langle B\left(u_{n}-u\right), u_{n}-u\right\rangle d t \\
&\left.\frac{1}{2}\left\langle B\left(u_{n}-u\right)(b),\left(u_{n}-u\right)(b)\right\rangle \geqslant 0 \text { (see hypothesis } H(B)\right) \\
& \Rightarrow \quad\left(\left(\hat{B} u, u_{n}^{\prime}-u^{\prime}\right)\right) \leqslant\left(\left(\hat{B} u_{n}, u_{n}^{\prime}-u^{\prime}\right)\right) \text { for all } n \in \mathbb{N} .
\end{aligned}
$$

Recall that

$$
\epsilon_{n}^{\frac{1}{2}}\left\|u_{n}\right\|_{L^{r}(T, X)} \leqslant M_{0} \text { for all } n \in \mathbb{N} \text { all } r \geqslant p \text { (see Proposition 5). }
$$

Suppose that $r_{m} \rightarrow+\infty, r_{m} \geqslant p$ for all $m \in \mathbb{N}$. Then for every $n \in \mathbb{N}, \epsilon_{n}^{\frac{1}{r_{m}}} \rightarrow 1$ as $m \rightarrow \infty$. Invoking Problem 1.175 of Gasinski and Papageorgiou [5], we can find $\left\{m_{n}\right\}_{n \geqslant 1}$ with $m_{n} \rightarrow+\infty$ such that

$$
\epsilon_{n}^{\frac{1}{r_{m_{n}}}} \rightarrow 1 \text { as } n \rightarrow \infty .
$$

Therefore there exists $n_{0} \in \mathbb{N}$ such that

$$
\begin{aligned}
& \frac{1}{2} \leqslant \epsilon_{n}^{\frac{1}{r_{n}}} \text { for all } n \geqslant n_{0}, \\
& \frac{1}{2}\left\|u_{n}^{\prime}\right\|_{L^{r_{m}}(T, X)} \leqslant M_{0} \text { for all } n \geqslant n_{0}, \\
\Rightarrow \quad & \left\|u_{n}^{\prime}\right\|_{L^{p}(T, X)} \leqslant 2 M_{0} \text { for all } n \geqslant n_{0}\left(\text { recall that } r_{m_{n}} \geqslant p\right) .
\end{aligned}
$$

On account of (15) and since $y=u^{\prime}$, we have

$$
u_{n}^{\prime} \stackrel{w}{\rightarrow} u^{\prime} \text { in } L^{p}(T, X) .
$$

Then from (26) and (27) it follows that

$$
0 \leqslant \liminf _{n \rightarrow \infty}\left(\left(\hat{B} u_{n}, u_{n}^{\prime}-u^{\prime}\right)\right)
$$

In addition, we have

$$
\epsilon_{n} \hat{K}_{p}\left(u_{n}^{\prime}\right) \rightarrow 0 \text { in } L^{p^{\prime}}\left(T, X^{*}\right) \text { as } n \rightarrow \infty \text { (see (18)). }
$$

By Proposition 5 and (27) it follows that

$$
\begin{aligned}
& \left\{u_{n}^{\prime}\right\}_{n \geqslant 1} \subseteq W_{p}(0, b) \text { is bounded, } \\
\Rightarrow \quad & \left\{u_{n}^{\prime}\right\}_{n \geqslant 1} \subseteq L^{p}(T, H) \text { is relatively compact (see (4)). }
\end{aligned}
$$


Therefore we have

$$
\begin{aligned}
& u_{n}^{\prime} \rightarrow u^{\prime} \text { in } L^{p}(T, H)(\text { see }(27)), \\
\Rightarrow & \left.\int_{0}^{b}\left(f_{n}, u_{n}^{\prime}-u^{\prime}\right) d t \rightarrow 0 \text { as } n \rightarrow \infty \text { (recall that } p \geqslant 2\right) .
\end{aligned}
$$

If in (24) we pass to the limit as $n \rightarrow \infty$ and use (25), (28), (29), (30), then

$$
\limsup _{n \rightarrow \infty}\left(\left(a\left(u_{n}^{\prime}\right), u_{n}^{\prime}-u^{\prime}\right)\right) \leqslant 0 .
$$

Invoking Theorem 2.35 of $\mathrm{Hu}$ and Papageorgiou [9, p. 41], we have

$$
a\left(u_{n}\right) \stackrel{w}{\rightarrow} a\left(u^{\prime}\right) \text { in } L^{p^{\prime}}\left(T, X^{*}\right) \text { as } n \rightarrow \infty .
$$

In (24) we pass to the limit as $n \rightarrow \infty$ and use (15) (with $v=u^{\prime \prime}$ ) (27), (29), (31). We obtain

$$
\begin{aligned}
& u^{\prime \prime}+a\left(u^{\prime}\right)+\hat{B} u=f, u(0)=u_{0}, u^{\prime}(0)=u_{1}, f \in S_{F\left(\cdot, u(\cdot), u^{\prime}(\cdot)\right)}^{2}, \\
\Rightarrow \quad & u \in S\left(u_{0}, u_{1}\right) \neq \emptyset .
\end{aligned}
$$

The proof is now complete.

3.1. An example. We illustrate the main abstract result of this paper with a hyperbolic boundary value problem. Let $\Omega \subseteq \mathbb{R}^{N}$ be a bounded domain. We consider the following boundary value problem

(32)

$$
\left\{\begin{array}{l}
\frac{\partial^{2} u}{\partial t^{2}}-\operatorname{div}\left(a(t, z)\left|D u_{t}\right|^{p-2} D u_{t}\right)+\beta(z) u_{t}-\Delta u=f(t, z, u)+\gamma u_{t} \text { in } T \times \Omega, \\
\left.u\right|_{T \times \partial \Omega}=0, u(0, z)=u_{0}(z), u_{t}(0, z)=u_{1}(z),
\end{array}\right\}
$$

with $u_{t}=\frac{\partial u}{\partial t}, 2 \leqslant p \leqslant \infty, \gamma>0$.

The forcing term $f(t, z, \cdot)$ need not to be continuous. So, following Chang [1], to deal with (32), we replace it by a multivalued problem (partial differential inclusion), by filling in the gaps at the discontinuity points of $f(t, z, \cdot)$. So we define

$$
f_{l}(t, z, x)=\liminf _{x^{\prime} \rightarrow x} f\left(t, z, x^{\prime}\right) \text { and } f_{u}(t, z, x)=\limsup _{x^{\prime} \rightarrow x} f\left(t, z, x^{\prime}\right) .
$$

Then we replace (32) by the following partial differential inclusion

$\left\{\begin{array}{l}\frac{\partial^{2} u}{\partial t^{2}}-\operatorname{div}\left(a(t, z)\left|D u_{t}\right|^{p-2} D u_{t}\right)+\beta(z) u_{t}-\Delta u \in\left[f_{l}(t, z, u), f_{u}(t, z, u)\right] \text { in } T \times \Omega, \\ \left.u\right|_{T \times \partial \Omega}=0, u(0, z)=u_{0}(z), u_{t}(0, z)=u_{1}(z) .\end{array}\right\}$

Our hypotheses on the data of (33) are the following:

$H(a): a \in L^{\infty}(T \times \Omega), a(t, z) \geqslant 0$ for almost all $(t, z) \in T \times \Omega$.

$H(\beta): \beta \in L^{\infty}(\Omega), \beta(z) \geqslant 0$ for almost all $z \in \Omega$.

$H(f): f: T \times \Omega \times \mathbb{R} \rightarrow \mathbb{R}$ is a function such that

(i) $f_{l}, f_{u}$ are superpositionally measurable (that is, for all $u: T \times \Omega \rightarrow \mathbb{R}$ measurable, the functions $(t, z) \mapsto f_{l}(t, z, u(t, z)), f_{u}(t, z, u(t, z))$ are both measurable);

(ii) there exists $a \in L^{2}(T \times \Omega)$ such that $|f(t, z, x)| \leqslant a_{2}(t, z)(1+|x|)$ for almost all $(t, z) \in T \times \Omega$, all $x \in \mathbb{R}$. 
Let $X=W_{0}^{1, p}(\Omega), H=L^{2}(\Omega)$ and $X^{*}=W^{-1, p^{\prime}}(\Omega)$. Then $\left(X, H, X^{*}\right)$ is an evolution triple with $X \hookrightarrow H$ compactly (by the Sobolev embedding theorem).

Let $A: T \times X \rightarrow X^{*}$ be defined by

$\langle A(t, u), h\rangle=\int_{\Omega} a(t, z)|D u|^{p-2}(D u, D h)_{\mathbb{R}^{N}} d z+\int_{\Omega} \beta(z) u h d z$ for all $u, h \in W_{0}^{1, p}(\Omega)$.

Then $A(t, u)$ is measurable in $t \in T$, continuous and monotone in $u \in W_{0}^{1, p}(\Omega)$ (hence, maximal monotone) and $\langle A(t, u), u\rangle \geqslant 0$ for almost all $t \in T$, all $u \in$ $W_{0}^{1, p}(\Omega)$.

Let $B \in \mathscr{L}\left(X, X^{*}\right)$ be defined by

$$
\langle B u, h\rangle=\int_{\Omega}(D u, D h)_{\mathbb{R}^{N}} d z \text { for all } u, h \in W_{0}^{1, p}(\Omega) .
$$

Clearly, $B$ satisfies hypothesis $H(B)$.

Finally, let $G(t, z, x)=\left[f_{l}(t, z, x), f_{u}(t, z, x)\right]$ and set

$$
F(t, u, v)=S_{G(t, \cdot, u(\cdot))}^{2}+\gamma v \text { for all } u, v \in L^{2}(\Omega) .
$$

Hypothesis $H(f)$ implies that $F$ satisfies $H(F)$.

Using $A(t, u), B u$ and $F(t, u, v)$ as defined above, we can rewrite problem (33) as the equivalent second order nonlinear evolution inclusion (1). Assuming that $u_{0} \in W_{0}^{1, p}(\Omega)$ and that $u_{1} \in L^{2}(\Omega)$, we can use Theorem 6 and infer that problem (30) has a solution $u \in C^{1}\left(T, L^{2}(\Omega)\right) \cap C\left(T, W^{1, p}(\Omega)\right)$ with $\frac{\partial u}{\partial t} \in L^{p}\left(\Omega, W_{0}^{1, p}(\Omega)\right)$ and $\frac{\partial^{2} u}{\partial t} \in L^{p^{\prime}}\left(\Omega, W^{-1, p^{\prime}}(\Omega)\right)$.

Note that if $a=0, f(t, z, x)=x$ and $\gamma=0$, then we have the Klein-Gordon equation. If $f(t, z, x)=f(x)=\eta \sin x$ with $\eta>0$, then we have the sine Gordon equation.

Acknowledgments. This work was supported by the Slovenian Research Agency grants P1-0292, N1-0064, J1-8131, and J1-7025, and the Romanian National Authority for Scientific Research and Innovation, CNCS-UEFISCDI, project number PN-III-P4-ID-PCE-2016-0130, within PNCDI III.

\section{REFERENCES}

[1] K.C. Chang, The obstacle problem and partial differential equations with discontinuous nonlinear terms, Comm. Pure Appl. Math. 33 (1980), 117-146.

[2] Z. Denkowski, S. Migórski, N.S. Papageorgiou, An Introduction to Nonlinear Analysis: Applications, Kluwer Academic Publishers, Boston, 2003.

[3] L. Gasinski, Existence of solutions for hyperbolic hemivariational inequalities, J. Math. Anal. Appl. 276 (2002), 723-746.

[4] L. Gasinski, N.S. Papageorgiou, Nonlinear Analysis, Chapman \& Hall/CRC, Boca Raton, FL, 2006.

[5] L. Gasinski, N.S. Papageorgiou, Exercises in Analysis. Part 1: Nonlinear Analysis, Springer, Heidelberg, 2014.

[6] L. Gasinski, M. Smolka, An existence theorem for wave-type hyperbolic hemivariational inequalities, Math. Nachr. 242 (2002), 79-90.

[7] L. Gasinski, M. Smolka, Existence of solutions for wave-type hemivariational inequalities with noncoercive viscosity damping, J. Math. Anal. Appl. 270 (2002), 150-164.

[8] S. Hu, N.S. Papageorgiou, Handbook of Multivalued Analysis. Volume I: Theory, Kluwer Academic Publishers, Dordrecht, The Netherlands, 1997.

[9] S. Hu, N.S. Papageorgiou, Handbook of Multivalued Analysis, Volume II: Applications, Kluwer Academic Publishers, Dordrecht, The Netherlands, 2000. 
[10] J-L. Lions, Quelques Méthodes de Résolution des Problèmes aux Limites Non Linéaires, Dunod, Paris, 1969.

[11] Z. Liu, S. Migórski, Noncoercive damping in dynamic hemivariational inequality with application to problem of piezoelectricity, Discrete Contin. Dyn. Syst. Ser. B 9 (2008), no. 1, 129-143.

[12] S. Migórski, Boundary hemivariational inequalities of hyperbolic type and applications, J. Global Optim. 31 (2005), 505-533.

[13] S. Migórski, Boundary hemivariational inequalities for a class of dynamic viscoelastic frictional contact problems, Computers Math. Appl. 52 (2006), 677-698.

[14] S. Migórski, A. Ochal, Vanishing viscosity for hemivariational inequalities modeling dynamic problems in elasticity, Nonlinear Anal. 66 (2007), no. 8, 1840-1852.

[15] A. Ochal, Existence results for evolution hemivariational inequalities of second order, Nonlinear Anal. 60 (2005), 1369-1391.

[16] N.S. Papageorgiou, V.D. Rădulescu, D.D. Repovš, Nonhomogeneous hemivariational inequalities with indefinite potential and Robin boundary condition, J. Optim. Theory Appl. $\mathbf{1 7 5}$ (2017), no. 2, 293-323.

[17] N.S. Papageorgiou, V.D. Rădulescu, D.D. Repovš, Sensitivity analysis for optimal control problems governed by nonlinear evolution inclusions, Adv. Nonlinear Anal. 6 (2017), no. 2, 199-235.

[18] N.S. Papageorgiou, N. Yannakakis, Second order nonlinear evolutions inclusions I: existence and relaxations results, Acta Math. Sinica (English Series) 21 (2005), 977-996.

[19] N.S. Papageorgiou, N. Yannakakis, Second order nonlinear evolutions inclusions II: structure of the solution set, Acta Math. Sinica (English Series) 22 (2006), 195-206.

[20] T. Roubiček, Nonlinear Partial Differential Equations with Applications, Birkhäuser, Basel, 2013.

[21] E. Zeidler, Nonlinear Functional Analysis and its Applications II/A and II/B, Springer, New York, 1990.

(N.S. Papageorgiou) National Technical University, Department of Mathematics, Zografou Campus, 15780 Athens, Greece \& Institute of Mathematics, Physics and MechanICS, 1000 LJubluana, Slovenia

E-mail address: npapg@math.ntua.gr

(V.D. Rădulescu) Institute of Mathematics, Physics and Mechanics, 1000 Luubljana, Slovenia \& Faculty of Applied Mathematics, AGH University of Science and Technology, 30-059 Kraków, Poland

E-mail address: vicentiu.radulescu@imfm.si

(D.D. Repovš) Faculty of Education and Faculty of Mathematics and Physics, UniverSity of Ljubljana, \& Institute of Mathematics, Physics and Mechanics, 1000 Ljubljana, SLOVENIA

E-mail address: dusan.repovs@guest.arnes.si 